\title{
Prevotella copri increases fat accumulation in pigs fed formula diets
}

Congying Chen

Shaoming Fang

Hong Wei

Maozhang He

Hao Fu

Xinwei Xiong

Yunyan Zhou

Jinyuan Wu

Jun Gao

Hui Yang

Lusheng Huang

\section{Video Byte}

Keywords: Microbiome, fat accumulation, lipid metabolism, digestion, metabolism, pigs, duroc pigs, gut microbiome, inflammation, metabolites, prevotella copri, bacterial community, formula diets, meat, pork, pigs, porcine, muscle growth, lipid transport, lipolysis, lipogenesis, mTOR signaling pathway, inflammatory response

Posted Date: October 13th, 2021

DOl: https://doi.org/10.21203/rs.3.rs-967260/v1

License: (9) This work is licensed under a Creative Commons Attribution 4.0 International License.

Read Full License 


\section{Abstract}

Excessive fat accumulation in pigs reduces economic returns in the pig industry. In humans and mice, the gut microbiome plays a role in host metabolism and fat accumulation, but the effects of a modern highenergy, high-protein diet on the gut microbiome and fat accumulation in pigs is not yet known. A recent study investigated the correlation between gut microbiome changes and fat accumulation in duroc pigs. They found that Prevotella copri abundance in the gut was positively associated with fat accumulation as well as increased obesity-related serum metabolite, increased host intestinal barrier permeability, and chronic inflammation. To examine causality, researchers isolated P. copri from pigs and administered it to germ-free mice. Treated mice showed similar phenotypes to pigs with high levels of P. copri, which included increased fat accumulation and altered serum metabolites. Their gene expression suggested increased chronic inflammatory response, lipid synthesis, and fat accumulation, but decreased lipid breakdown, lipid transport, and muscle growth. These results suggest that P. copri activates the host chronic inflammatory response and fat accumulation as well as highlight a potential target to reduce fat accumulation in pigs. 